\title{
Preliminary SuperTIGER Abundances of Galactic Cosmic-Rays for the Charge Interval $Z=41-56$
}

\author{
N.E. Walsh*, W.R. Binns, M.H. Israel, R.P. Murphy, B.F. Rauch, J.E. Ward \\ Washington University \\ St. Louis, MO 63130, USA \\ E-mail: newalsh@wustl.edu
}

T.J. Brandt, J.T. Link, J.W. Mitchell, T. Hams, K. Sakai, M. Sasaki

NASA Goddard Space Flight Center

Greenbelt, MD 20771, USA

\author{
A.W. Labrador, R.A. Mewaldt, E.C. Stone \\ Stone California Institute of Technology \\ Pasadena, CA 91125, USA
}

\section{M.E. Wiedenbeck}

Jet Propulsion Laboratory, California Institute of Technology

Pasadena, CA 91125, USA

\section{C.J. Waddington}

University of Minnesota

Minneapolis, MN 55455, USA

\begin{abstract}
On December 8, 2012 the SuperTIGER (Super Trans-Iron Galactic Element Recorder) instrument was launched from Williams Field, Antarctica on a long-duration balloon flight that lasted 55 days and maintained a mean altitude of 125,000 feet. SuperTIGER measured the relative abundances of Galactic cosmic-ray nuclei with high statistical precision and well resolved individual element peaks from Neon to Zirconium (charges $Z=10-40$ ). SuperTIGER also made exploratory measurements of the relative abundances up to Barium $(Z=56)$. Although the statistics are low for elements heavier than Zirconium, we will show how the relative abundances of charges $Z=41-56$ compare to those reported by HEAO3-HNE during 1979-81. The relative abundances of elements Zirconium through Neodymium ( $\mathrm{Z}=60)$ are of particular interest because they are likely formed both by supernova explosions and by binary neutron star mergers. A well resolved measurement of this range of elements can constrain the contributions to the Galactic cosmic-ray composition from both of these possible sources.
\end{abstract}

36th International Cosmic Ray Conference -ICRC2019-

July 24th - August 1st, 2019

Madison, WI, U.S.A.

${ }^{*}$ Speaker. 


\section{Introduction}

The SuperTIGER (ST) instrument has undergone minor improvements and refurbishments since its first, 55 day flight (ST-I) in December, 2012 (Figure 1, Binns et al., 2014) and is planned to launch from McMurdo Station, Antarctica on a second long-duration balloon flight (ST-II) during the Austral summer of 2019-20. The instrument design is focused on making well resolved individual element abundance measurements of ultra-heavy Galactic cosmic rays (UHGCR). The data produced is expected to improve the statistics of existing ST-I measurements made in the charge range $Z=30-40$ and to allow the first accurate measurements of individual elements in the charge range $\mathrm{Z}=40-56$.

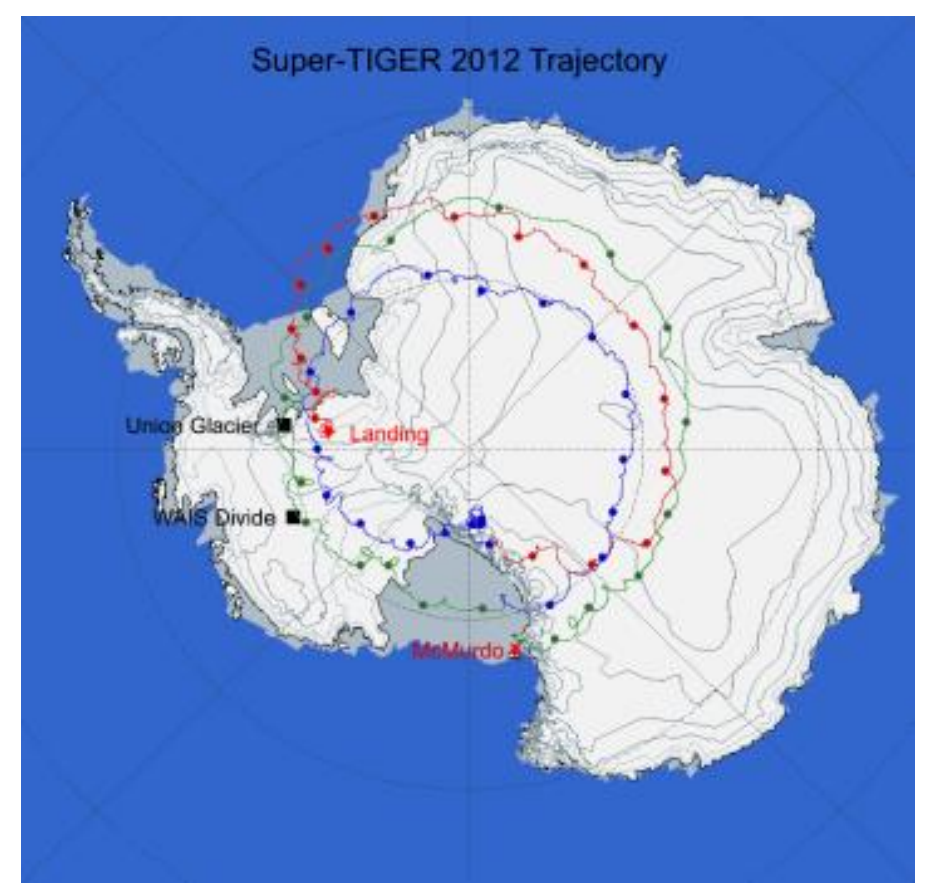

Figure 1: SuperTIGER 2012 flight path. Completed 2.75 rotations about the South Pole.

ST-I collected excellent data on over 5 million GCR ${ }_{26} \mathrm{Fe}$ and heavier nuclei (about 44 equivalent days of data) and ST-II is hoped to achieve a similar number of events. The ST-II flight will occur during solar minimum, where a $\sim 50 \%$ increase in the cosmic-ray flux is expected over that of ST-I. If ST-II flies for 30 days then the amount of data collected is expected to be equivalent to $\sim 44$ days of data collected by ST-I.

The data analysis method used for ST-II will be similar to that utilized for the ST-I flight. When data from the two flights are combined we can expect sufficient improvement in the statistics such that the elements greater than ${ }_{40} \mathrm{Zr}$ can be measured with improved precision. The prospects for this measurement can be shown by comparing the exploratory ST-I Z>40 measurements with those made in this range by the Heavy Nuclei Experiment aboard the third High-Energy Astronomy Observatory satellite (HEAO-3-HNE). 


\section{Instrument Overveiw}

The ST-I instrument was recovered from Antarctica during the Austral summer of 2014-15 and was found to be in overall excellent condition. It was decided that the original instrument could be flown again for a second flight, ST-II, and so the ST-I\&II instrumentation is essentially identical, save for some minor refurbishments and improvements. In addition, the high voltage power supply and on-board data recording system have been redesigned. Since the 2014-15 recovery, ST-II has had two unsuccessful flight seasons. In 2017-18, the weather provided undesireable flight conditions and so a flight was not attempted. In 2018-19, a flight was attempted but after several hours, the balloon decended and ST was recovered in the same season. We are scheduled for a third season this Austral summer, 2019-20.

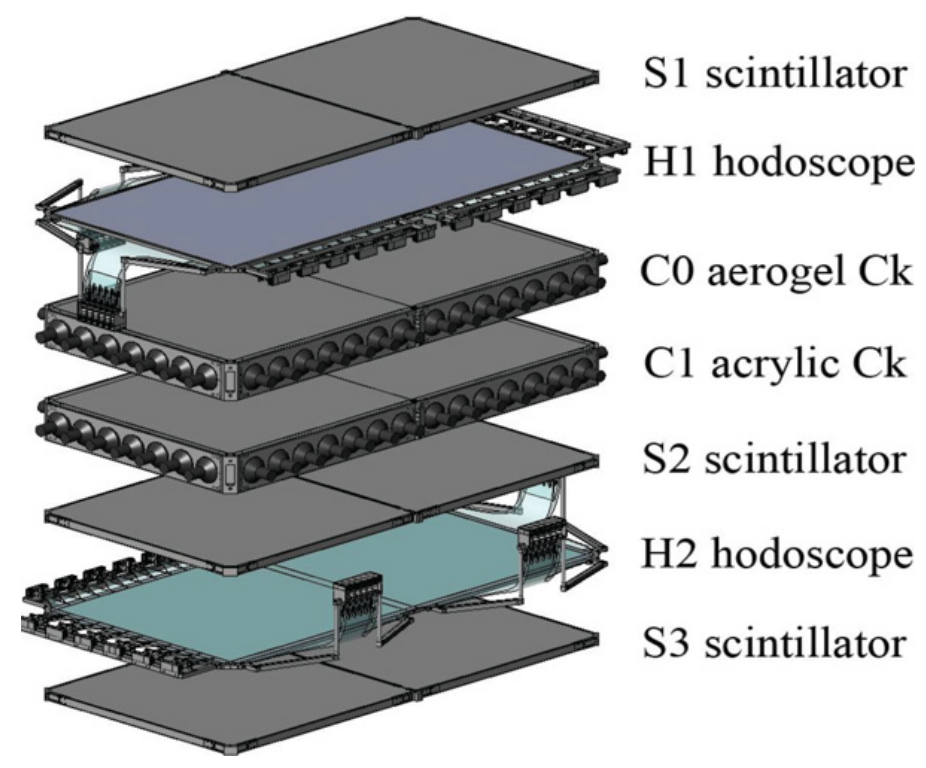

Figure 2: SuperTIGER detector stack. One module shown.

The instrument consists of two nearly identical modules, each containing seven stacked detectors (Figure 2, Binns et al., 2014). From top to bottom, in each module there is a plastic scintillation detector (S1), a scintillating optical fiber hodoscope plane (H1), a silica- aerogel Cherenkov detector (C0), an acrylic Cherenkov detector (C1), a second scintillation detector (S2), a second hodoscope plane (H2), and a third scintillation detector (S3). The signals from the S and C detectors are used in combination to measure both the charge and kinetic energy of cosmic rays that pass through the entire stack. The H layers are used to determine each cosmic ray's trajectory through the instrument, allowing angle corrections and area mapping to be performed.

The SuperTIGER instrument is designed to measure UHGCR, which are both rare and interact readily, so maximizing statistics and minimizing particle interactions are priorities. To achieve these priorities, maximum altitude and minimum interactions are attained by building the instrument with light weight structural materials and with as little material in the beam line as possible. High statistics are acheived by the instrument's large acceptance geometry. Each module has an active area of $1.16 \mathrm{~m}$ by $2.4 \mathrm{~m}$ and together give the instrument a combined effective geometry 
factor of $3.9 \mathrm{~m} 2 \mathrm{sr}$ (after interactions are removed using ${ }_{34} \mathrm{Se}$ nuclei as a reference) (Binns et al., 2014).
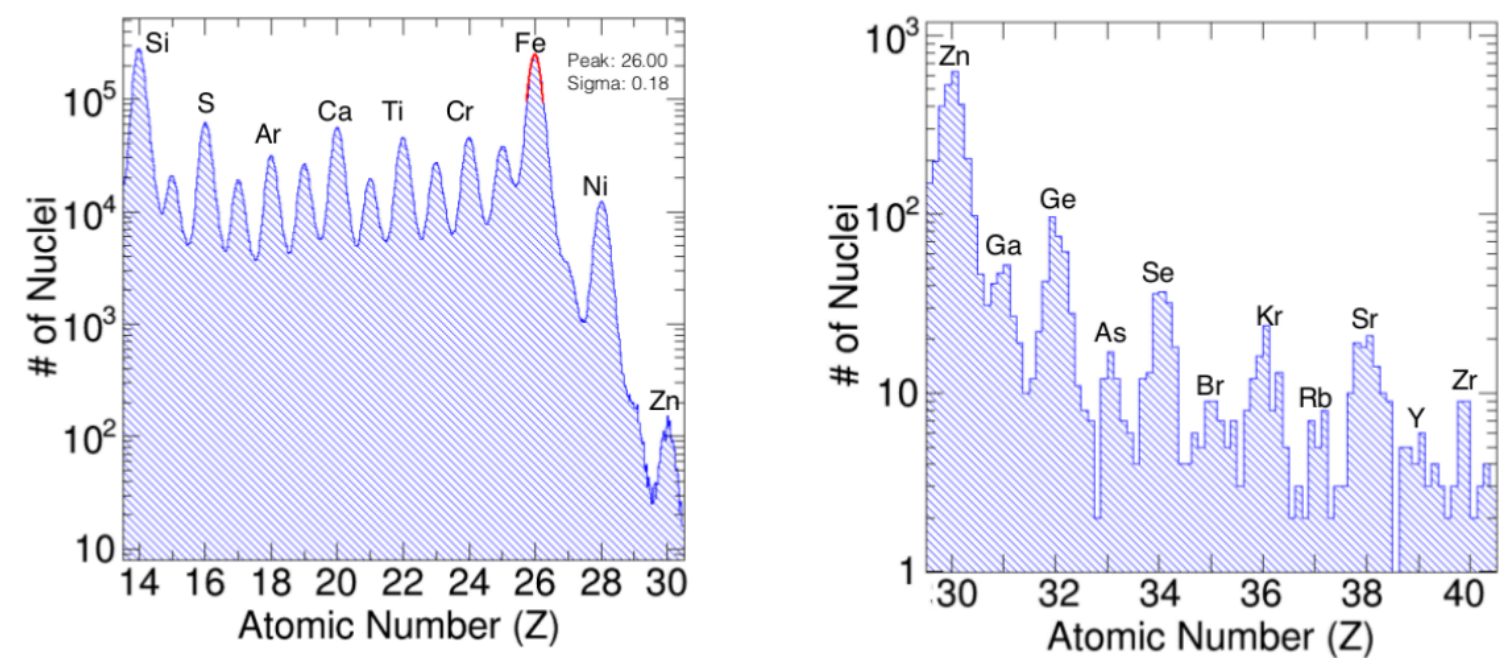

Figure 3: Left: SuperTIGER $Z=14-30$ elemental abundances. Individual element peaks are clearly visible. Right:SuperTIGER Z=30-40 elemental abundances. Individual element peaks are visible for entire charge range.

\section{SuperTIGER $\mathrm{Z}=\mathbf{3 0}-\mathbf{4 0}$}

ST-I made the best UHGCR abundance measurements to date in the charge range Z=30-40 (Murphy et al., 2016). Individual element peaks are clearly resolved from ${ }_{14} \mathrm{Si}$ to ${ }_{30} \mathrm{Zn}$ (aside from ${ }_{27} \mathrm{Co}$ and ${ }_{29} \mathrm{Cu}$ ) with the ${ }_{26} \mathrm{Fe}$ peak having a standard deviation $(\sigma)$ of $0.18 \mathrm{cu}$ (Figure $3 \mathrm{Left}$ ). Although the statistics are much lower, individual element peaks are also well resolved from ${ }_{30} \mathrm{Zn}$ to ${ }_{40} \mathrm{Zr}$ (Figure 3 Right).

When plotting the Galactic-cosmic-ray-source (GCRS) abundances relative to the solar system (SS) abundances (Lodders, 2003), patterns for the preferential acceleration of refractory elements (dust) over the volatiles (gas) and for a mass dependence in GCR volatile acceleration cannot be clearly discerned (Figure 4 Left). When instead the GCRS abundances are plotted relative to a mixture of $\sim 80 \%$ SS material with $\sim 20 \%$ massive star material (MSM) the refractories and volatiles separate completely and the mass trend of the acceleration of both the refractories and volatiles emerges (Figure 4 Right). The ST results combined with those from TIGER (Rauch et al., 2009) and HEAO-3-C2 (Engelmann et al., 1990) show that refractories are accelerated preferentially over volatiles by a factor of $\sim 4$ and that the GCR source material is a mixture of massive star material and normal interstellar medium (ISM) material.

To achieve excellent charge resolution over wide charge and energy ranges, two complementary methods were used in the data analysis. The first method utilizes the signals from the two Cherenkov detector layers $\mathrm{C} 0$ and $\mathrm{C} 1$. The $\mathrm{C} 0$ material, aerogel, has an index of refraction $\mathrm{n}=1.04$ $(\mathrm{n}=1.025$ in one half module) while the $\mathrm{C} 1$ material, acrylic, has an index $\mathrm{n}=1.49$. Since the Cherenkov signal only occurs when the particle velocity exceeds $\beta=1 / \mathrm{n}$, this method can only be used for particles with energies greater than $2.5 \mathrm{GeV} /$ nucleon (or $3.3 \mathrm{GeV} /$ nucleon for $\mathrm{n}=1.025$ ), 

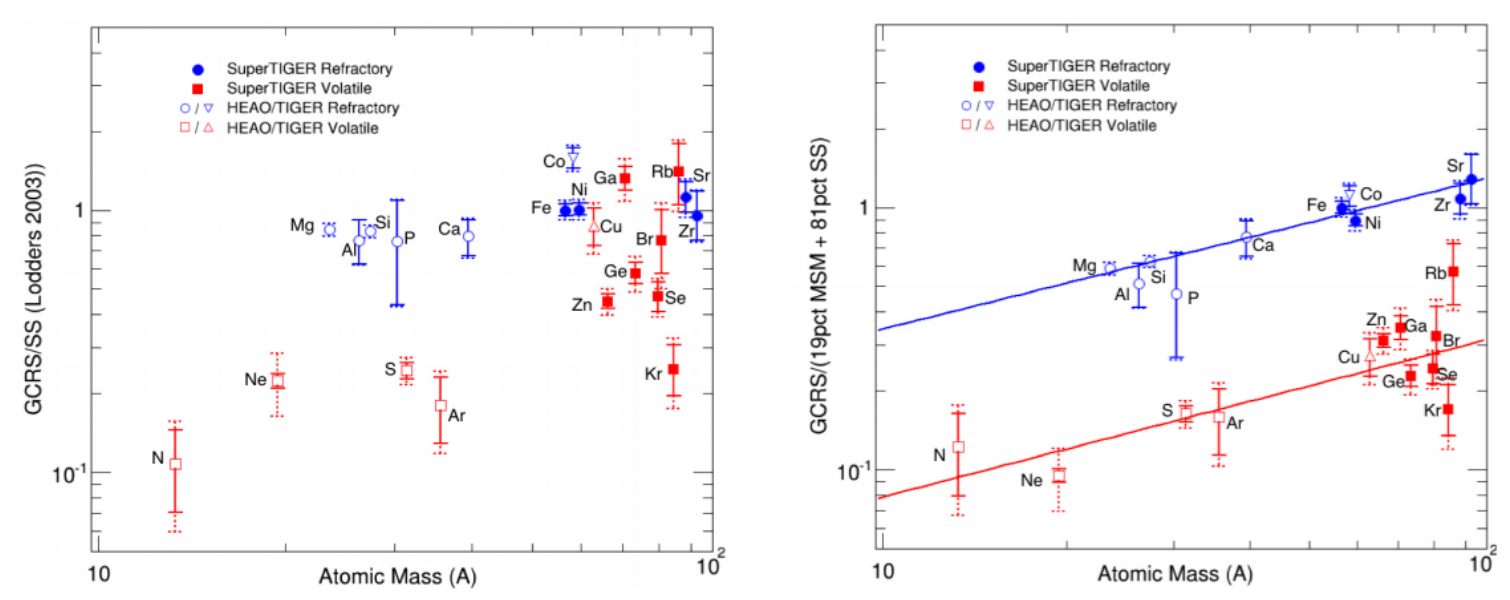

Figure 4: Left: Dependence on A of GCRS material relative to SS. Refractories and volatiles not clearly separated for high mass nuclei. Right: Dependence on A of GCRS material relative to mixture of MSM with normal ISM (SS). Refractories preferentially accelerated over volatiles by factor of $\sim 4$.

where the $\mathrm{C} 0$ detector "turns on". Even though the charge determination made via the combined Cherenkov method yields better charge resolution, for lower energies, where $\mathrm{C} 0$ does not give a Cherenkov signal, a method using a combination of scintillation detector signals with the $\mathrm{C} 1$ signal must be used. This method works down to energies of $\sim 350 \mathrm{MeV} /$ nucleon, where the $\mathrm{C} 1$ detector turns on, which is sufficiently low to include most UHGCR that pass through the instrument.

These complementary charge identification methods can be illustrated with cross plots of S1 vs. C1 (Figure 5 Left) for energies below the $\mathrm{C} 0$ threshold, and $\mathrm{C} 1$ vs. C0 signals (Figure 5 Right) for energies above the $\mathrm{C} 0$ threshold. One can easily see the charge bands that emerge when plotting the signals in this fashion, and it is the clear separation between these bands that can be used to determine charge for particles passing through the instrument. The complementary nature of these methods is clear when comparing the "Above" and "Below"-C0 regions in these two plots. An approximation of the $\mathrm{C} 0$ threshold energy line is drawn in red on both the $\mathrm{C} 1 \mathrm{vs}$. $\mathrm{C} 0$ and the $\mathrm{S} 1$ vs. $\mathrm{C} 1$ plots, and it can be seen that, although the Above- $\mathrm{C} 0$ region to the right of the line is very dense on the S1 vs. C1 plot, the particles in the same energy region to the right of the line on the $\mathrm{C} 1$ vs. $\mathrm{C} 0$ plot spread out nicely into charge bands. The Below-C0 region is similarly dense on the left of the line in the $\mathrm{C} 0$ vs. $\mathrm{C} 1$ plot and is resolved on the left of the line in the $\mathrm{S} 1 \mathrm{vs}$. $\mathrm{C} 1$ plot.

For the Above- $\mathrm{C} 0$ method ( $\mathrm{C} 1$ vs. $\mathrm{C} 0$ ), charge determination is fairly straightforward. In the analysis of Murphy et al. (2016) a second order polynomial was fit to the ${ }_{26} \mathrm{Fe}$ charge band for several different angle bins. The Cherenkov light produced by a charged particle (and therefore the Cherenkov detector signal) is proportional to $\mathrm{Z} 2$, so that the parallel $\mathrm{C} 1$ vs. $\mathrm{C} 0$ charge bands are separated by such a factor. If one then finds the functional form of one line (the iron line in this case) one can solve for the charge of any Above- $\mathrm{C} 0$ particle from its $\mathrm{C} 1$ and $\mathrm{C} 0$ detector signals.

The Below-C0 method ( $\mathrm{S}$ vs. $\mathrm{C} 1$ ) is a more complicated procedure as the scintillation light produced by a charged particle does not have a straightforward dependence on the charge. To achieve better charge resolution, the analysis is done separately for both S1 vs. C1 and for S2 vs. $\mathrm{C} 1$ and then the charge determination from both methods is averaged. For different angle bins, the charge bands are fit with second order polynomials. In addition, lines of constant energy are plotted 

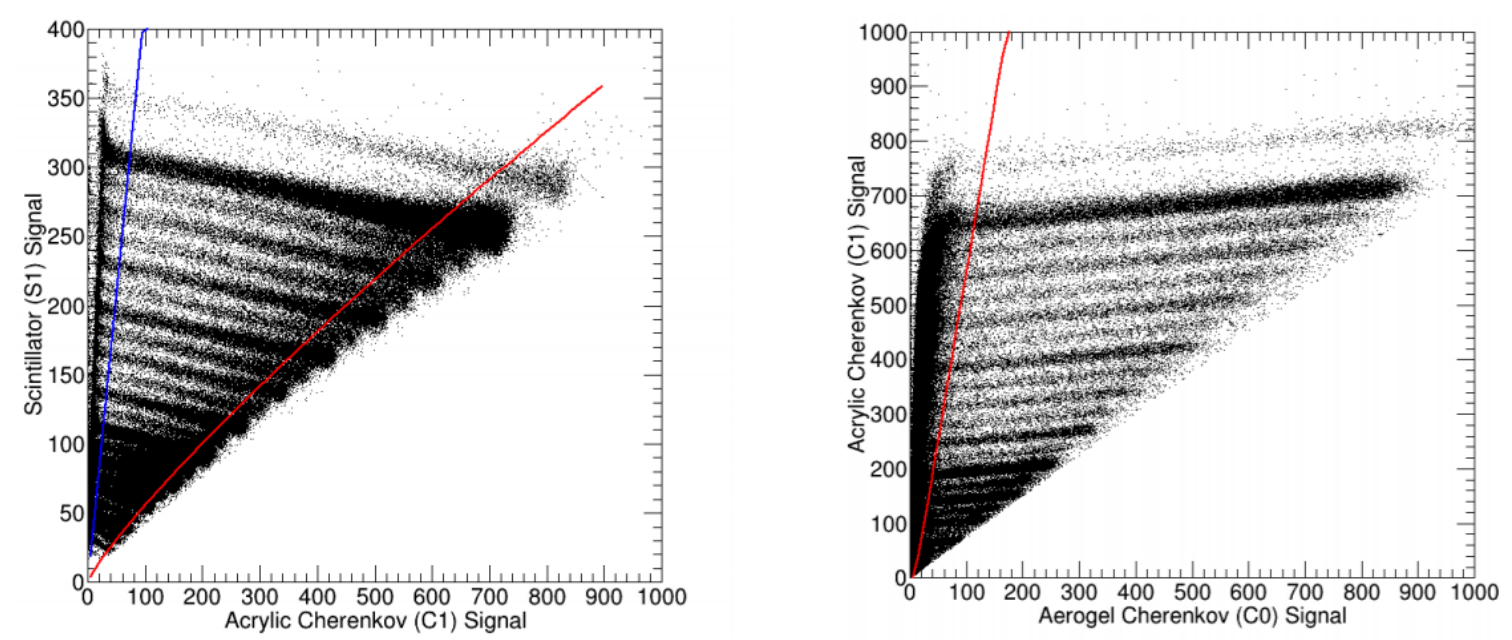

Figure 5: Left: Below-C0 method ( $\mathrm{S} 1$ vs. $\mathrm{C} 1$ ). Blue line is low energy cut. Red line is approximate $\mathrm{C} 0$ threshold energy. Particles to the left are well resolved. Right: Above-C0 method (C1 vs. C0). Red line is approximate $\mathrm{C} 0$ threshold energy. Particles to the right are well resolved.

which span the $\mathrm{S}$ vs. C1 space. The intersections between the charge contours and lines of constant energy can be found, and the scintillator signal as a function of charge for each energy can be fit with a model of scintillation light yield (Murphy used the Voltz model). Finding the functional parameters of each fit reveals the dependence of these parameters on energy. Once this dependence is known, the fitting function can be solved for $\mathrm{Z}$ for any particle. Relating particle energy to the $\mathrm{C} 1$ signal makes it possible to solve for Below- $\mathrm{C} 0$ particle charges from $\mathrm{S}$ and $\mathrm{C} 1$ signals.

The charges are determined for events from the Above and Below-C0 energy regions with these methods. Interaction cuts and velocity corrections appropriate to each of these energy regions are applied and Above and Below-C0 charge determinations are finalized. The resulting combined Above and Below-C0 charge histograms (Figure 3 Left \& Right) have well resolved peaks.

\section{SuperTIGER $\mathrm{Z}=\mathbf{4 1 - 5 6}$}

In order to analyze the UHGCR with charge higher than ${ }_{40} \mathrm{Zr}$, the above method for charge determination must be modified as it is optimized for the $\mathrm{Z}=30-40$ range. In order to see charge peaks up to ${ }_{56} \mathrm{Ba}$, the analysis must prioritize statistics by including as much data as possible without compromising the resolution. Several improvements to Murphy's analysis were made to achieve this. First, the solid state drives that were recovered after the initial analysis supplied $\sim 5$ days of additional data. Although most of the new events were low priority data that was not telemetered, our statistics are improved in the high charge regime after its addition. Second, the analysis of the ST scintillator response during a CERN beam run test, showed that the Tarle (or BTV) model (Tarle et al., 1979) for scintillator saturation was a better fit than the Voltz model (Voltz et al., 1966) for high charges (M. Sasaki), so this saturation model was employed in the new analysis. Additionally, in the previous analysis, the saturation model parameters were found for each of 30 angle and 50 energy bins and then a simple interpolation was done between angle and energy bins separately to determine how the saturation model parameters varied between bins. In the new analysis, we 
employ bilinear interpolation over angle and energy bins simultaneously to find the best estimate of the charge given its four nearest angle and energy bins. Finally, the interaction cuts applied to achieve the desired resolution on the $\mathrm{Z}=30-40$ range are loosened carefully to include more particles without hurting the resolution. Heavier particles undergo interactions much more readily and therefore our data lacks the statistics that would allow for harsh cuts in the high $\mathrm{Z}$ regime. Once the cuts are loosened, the preliminary Above and Below-C0 combined histogram features charge peaks all the way up to $\mathrm{Z}=60$ (Figure $6 \mathrm{Left}$ ) and a side by side comparison to HEAO-3-HNE data (Figure 6 Right, Binns et al., 1989) can be made.
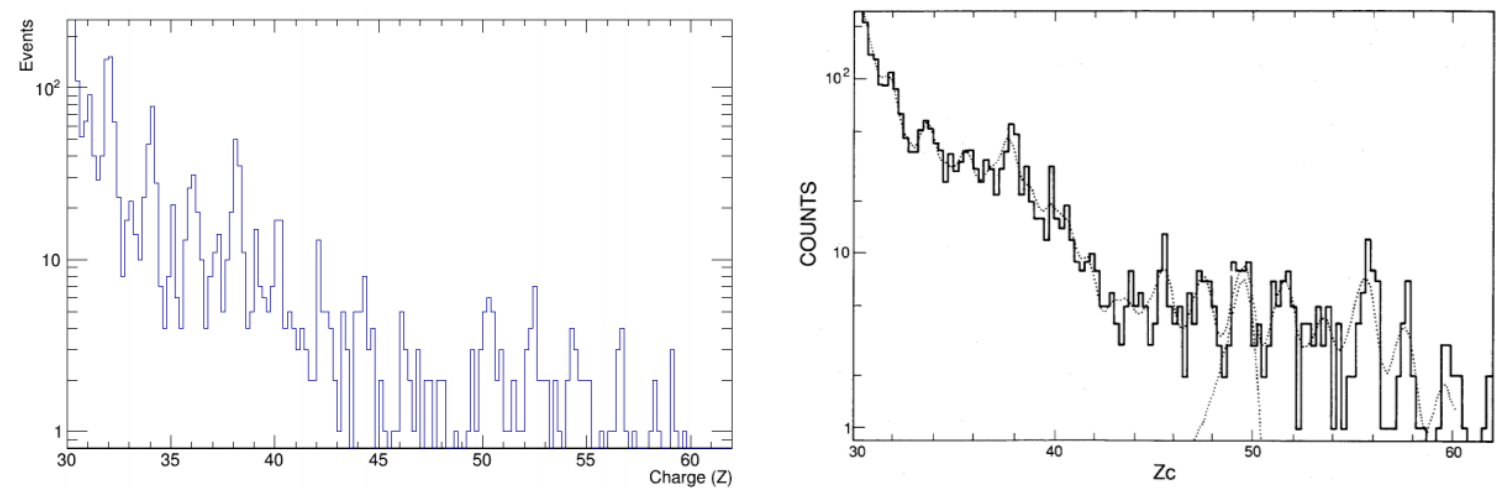

Figure 6: Left: Preliminary, instrument altitude, ST elemental abundances for $\mathrm{Z}=30-60$. Individual element peaks visible. Peaks do not land on the integers. A secondary energy correction is still needed. Right: HEAO-3-HNE elemental abundances for $\mathrm{Z}=30-60$. Resolution was inadequate to distinguish odd peaks.

Comparing the ST balloon-altitude measurements with the HEAO-3 space-based measurements requires additional analysis. The ST instrument abundances must be corrected for atmospheric nuclear interactions and energy losses to give top-of-the- atmosphere (TOA) abundances.

\section{Impact of Solar Modulation}

After comparing ST-I elemental abundances with those of HEAO-3-HNE it is clear that a second flight (ST-II) will significantly improve the measurements above $\mathrm{Z}=40$. Flying an almost identical instrument will provide equally well resolved charge determinations that will add to the existing peaks from ST-I and make them more well defined. SuperTIGER achieved a 55 day flight and, due to the necessity to rely on telemetered data, effectively 44 days of measurement were analyzed by Murphy et al. in 2012-13. In this time, it was able to measure UH nuclei corresponding to more than 5 million GCR Fe nuclei. In the 2019-20 Austral summer ST- II will fly during solar minimum when there will be lower solar modulation, allowing more GCR events per unit time to be collected (Figure 7, R.A. Mewaldt). In 2012-13, during the ST-I flight, the solar modulation was $\sim 540 \mathrm{MV}$, blocking 43\% of the GCR flux seen in the 2009-10 solar minimum with a modulation factor of $\sim 250 \mathrm{MV}$. If the modulation reaches $250 \mathrm{MV}$ as in 2009 , we expect to measure $\sim 43 \%$ more GCR Fe per unit time. So it is very reasonable to assume a $50 \%$ increase in the cosmic-ray flux we will see with ST-II over ST-I. Thus, ST-II will only need to fly for 30 days to measure the same number of particles that ST-I did in 2012-13. 


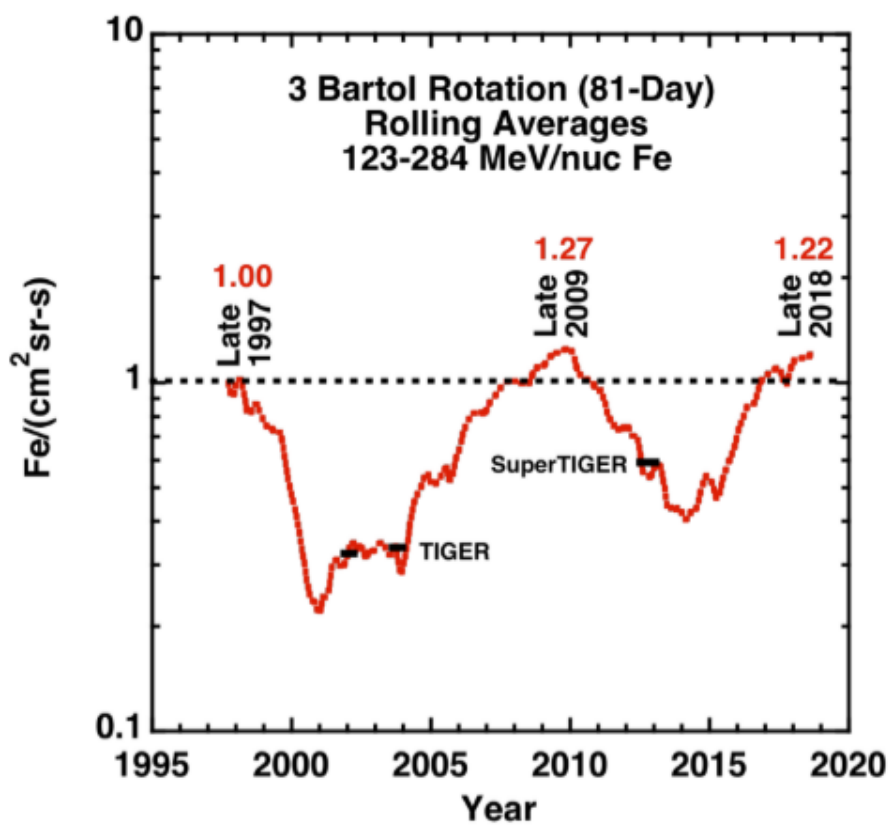

Figure 7: Cosmic-ray Fe flux changing through solar cycle. ST-I flew 2012-13 and ST-II will fly 2019-20. Cosmic-ray flux will be much higher for ST-II than for ST-I.

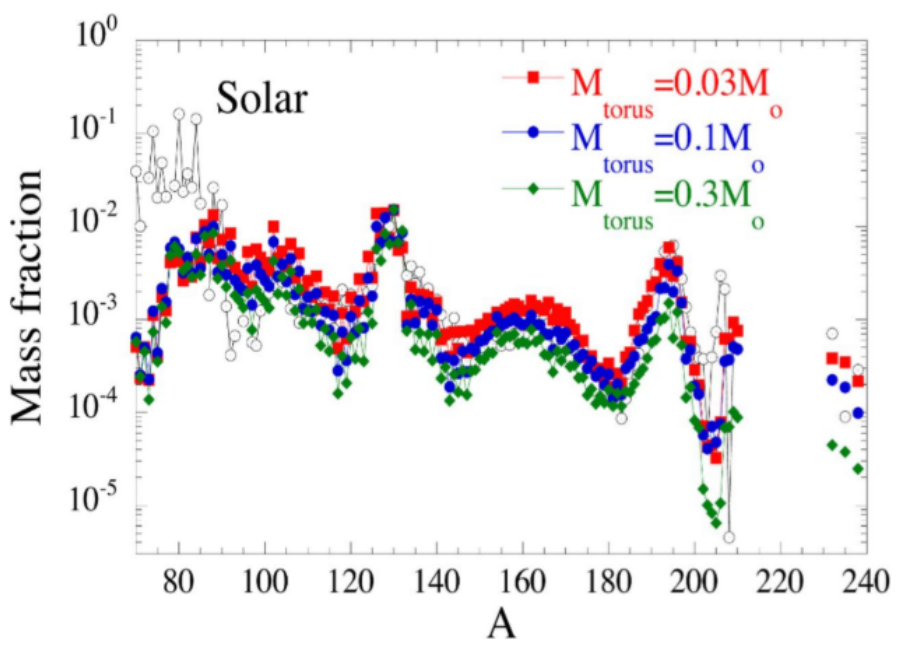

Figure 8: Some models suggest that all of the r-process nuclei could be produced by BNSM.

\section{Conclusion}

ST-II will increase our total ST statistics and allow us to make more accurate abundance measurements of the UHGCR that could yield more information about the origins of GCR. Well resolved easurements above $\mathrm{Z}=40$ would allow us to add additional points to Figure 4 Right, increasing the lever arm on the mass trend into a higher mass region. ST-I data showed that an $\sim 80 \%$ SS to $\sim 20 \%$ MSM mixture made sense of the refractory over volatile preferential acceleration in the GCR, and pointed to GCR having a significant origin in OB associations. However, with more 
points to add in the higher mass region we may see changes in this plot that point to other GCR sources, such as binary neutron star mergers (Figure 8, Just et al., 2015). In particular, we would like to place constraints on the production models of supernovae and binary neutron star mergers.

\section{References}

[1] W.R. Binns et al., The SuperTIGER Instrument: Measurements of Elemental Abundances of Ultra-Heavy Galactic Cosmic Rays, ApJ 788 (2014) 18-28.

[2] R.P. Murphy et al., Galactic Cosmic Ray Origins and OB Associations: Evidence from SuperTIGER Observations of Elements 26 Fe Through $40 \mathrm{Zr}$, ApJ 831 (2016) 2083-2088.

[3] K. Lodders, Solar System Abundances and Condensation Temperatures of the Elements, ApJ 591 (2003) 1220-1247.

[4] B.F. Rauch et al., Cosmic Ray origin in OB Associations and Preferential Acceleration of Refractory Elements: Evidence from Abundances of Elements 26 Fe through 34 Se, ApJ 697 (2009) 2083-2088.

[5] J.J. Engelmann et al., Charge composition and energy spectra of cosmic-ray nuclei for elements from Be to NI - Results from HEAO-3-C2, A\&A 233 (1990) 96-111.

[6] W.R. Binns et al., Abundances of Ultraheavy Elements in the Cosmic Radiation: Results from HEAO 3, ApJ 346 (1989) 997-1009.

[7] R. Voltz et al., Influence of the Nature of Ionizing Particles on the Specific Luminescence of Organic Scintillators, JChPh 45 (1966) 3306.

[8] G. Tarle et al., Cosmic Ray Isotope Abundances from Chromium to Nickel, ApJ 230 (1979) 607-620.

[9] O. Just et al., Comprehensive Nucleosynthesis Analysis for Ejecta of Compact Binary Mergers, Monthly Notices of the Royal Astronomical Society 000 (2015) 1-30. 